\title{
Theoretical and experimental study on active vibration control of smart
}

\section{structures}

\author{
Zhou Li
}

Automotive Engineering institute, Jiangxi University of Technology, Nanchang 330098, China

Keywords: Active vibration control; Smart structures; Independent modal space control

\begin{abstract}
The application of intelligent material systems and structures is very extensive, which is not only in sophisticated weapons for national defense such as aircraft, ships, but also in various fields of the national economy. They are of great strategic significance especially in the field of high technology. Currently the main materials of intelligent material systems and structures include shape memory materials, piezoelectric materials (including piezoelectric ceramics and piezoelectric polymer), electrostrictive material, optical fiber and the current variant and magnetic current variant, etc. A material system and structure integrating driving, sensing and controlling can be achieved by combining the use of these smart materials' function, with ingenious and exquisite composite design and fabrication. This paper adopts the finite element method that is widely used in engineering to model the plate structure. It analyzes the finite element and implements numerical simulation research on the active vibration control.
\end{abstract}

\section{Introduction}

With the development of high-tech and industry, the structure develops towards large scale and the vibration environment is more complex, which means that using traditional structural dynamics for design will be harder. Structure vibration control has become one of the major issues of modern engineering technology. The intelligent control to inhibit the deformation and dynamic disturbance of structure has been paid high attention by engineering and theory circles.

The active vibration control of structure is to use external energy to provide control to reduce the structural response under environmental loads, which is considered to be an effective method. Active vibration control is a research field combining vibration theory and modern control theory. The research object is a closed loop system composed of controlled structure and control structure, called active vibration control system. The system relies on the structural vibration feedback to be able to adapt to unpredictable external interference and uncertainty of the structure flexibly, which has a strong ability to adapt to the environment and suppress the low-frequency vibration and broadband random vibration. Active damping control has these advantages to make up for the shortcomings of passive control of structure, which has been widely studied.

In recent years, the research on structural vibration control has attracted more and more attention and structure control research has become a hot subject of structural analysis. Because of the development of computer technology nowadays, the high speed, large storage and strong graphic function of the computer has laid the material foundation for the simulation of computer structural vibration. On the other hand, the high cost of the experiment and more and more real-time computing needs of the active control technology make the researchers use the computer to simulate the effect 
of the control, so the application of simulation technology has become inevitable. Because the beam slab structure is a relatively common and main structure form, the simulation research of beam plate structure has played an important role in further research of other more complex structures.

In decades, with the rapid development of material science, control, microelectronics and computer technology, especially the breakthrough of research on new sensors and actuators, in the active control of structural design, the new sensing and actuating material replaced the conventional sensors and actuators, promoting the development of new technology in the structure design and generating smart structure, a new form of structure.

The application of intelligent material systems and structures is very extensive, not only in sophisticated weapons for national defense such as aircraft, ships, but also in various fields of the national economy. They are of great strategic significance especially in the field of high technology. Currently the main materials of intelligent material systems and structures include shape memory materials, piezoelectric materials (including piezoelectric ceramics and piezoelectric polymer), electrostrictive material, optical fiber and the current variant and magnetic current variant, etc. A material system and structure integrating driving, sensing and controlling can be achieved by combining the use of these smart materials' function, with ingenious and exquisite composite design and fabrication.

In the dynamic modeling of piezoelectric structure, the construction of the dynamic model of the piezoelectric structure is a foundation for the control of the piezoelectric structure, mainly aiming to establish correspondence between the mechanical properties and electrical properties of the structure, namely, the relationship between output and structure of sensing elements (sensing equation), the effect of the input of driving elements on the structure (the driving equation), and the study on the deformation and motion of the whole structure. There are 3 kinds of commonly used modeling methods: analytical modeling, numerical modeling and experimental modeling.

The computer simulation technology is a comprehensive science and technology developed in recent thirty years. The more popular definition of simulation is that "simulation" is the process to use model to test and research the actual system [13]. According to the type of model, simulation is usually divided into two categories: physical simulation and mathematical simulation. If a physical model is used, it is called physical simulation. If a mathematical model is used, namely, the use of related principles in the disciplines and some experimental data to construct the mathematical model, then the "solution" of the mathematical model is called mathematical simulation. The computer can be conveniently used to solve the mathematical model, so the computer is the main tool of mathematical simulation. Nowadays, the development of the computer technology, the high cost of experiment and the better active control technology enable researchers to use computers to simulate the vibration control effect increasingly commonly, so the application of simulation technology has become inevitable.

This paper utilizes finite element method that is widely used in engineering to model the plate structure, analyzes the finite element and conducts numerical simulation research on its active vibration control. Finally, it uses the example of the board to verify the effectiveness of the former works.

\section{Methods}

When the piezoelectric material is subjected to mechanical deformation, relative movement occurs in the internal positive and negative charge center of the material and polarization is generated, 
resulting in two bound charges of opposite sign on the surface of the element and the charge density being proportional to the external force. This phenomenon is called positive piezoelectric effect. It reflects that the piezoelectric materials have the ability to convert mechanical energy into electrical energy. If the change of electrical charge of piezoelectric element is detected, we can know the deformation quantity of the structural components or the position elements are embedded in, so the piezoelectric effect is used to make the piezoelectric materials into the sensing element.

If the two faces of the piezoelectric element are charged the voltage, due to the effect of electric field, the internal positive and negative charge center of the piezoelectric element is made to produce relative displacement, leading to deformation of the piezoelectric elements. This phenomenon is called the inverse piezoelectric effect. The inverse piezoelectric effect reflects that piezoelectric materials have the ability to transfer the electric energy into mechanical energy. The inverse piezoelectric effect is used to make piezoelectric materials into pneumatic components.

Because the piezoelectric material has positive piezoelectric effect and inverse piezoelectric effect, not only can it be used as actuators and sensors, but it also is often attached to the surface of the main structure or embedded in it to form a piezoelectric active structure. The practice proves that the piezoelectric active control has the advantages of high efficiency and self-adaptability, having aroused great interest in recent years.

Piezoelectric ceramic is a kind of functional material with the characteristics of being deformed by electricity. Although the deformation is small, it needs strong electric field and great stress to limit the deformation, so it is a good micro actuator. According to the requirements of structural vibration damping, the expansion deformation of piezoelectric ceramics is used to change the fastening force of the friction plate so as to adjust Mount relay size in real time, which can make the friction dampers have intelligent characteristics and good prospects for application development.

Because the board structure is a simple and universal structure, the study on the modeling is representative. But the piezoelectric laminated plate is a structure of 3 layers with the elastic material as well as nonlinear materials, so the finite element modeling and model analysis are the key technology.

Since the 70's when finite element technology came into being, many mechanical problems that cannot be solved or cannot thoroughly be solved in the past can be solved using this technology. Over the past 30 years the finite element technology is already so mature that there is no mechanical problem that cannot be solved with finite element. But as for the laminated unit, a finite element model shall be constructed to analyze it. When it is necessary, compare and analyze different units used to solve the same problem. The shortcoming of the application of this method is that it is difficult to model complex structure. The more accurate the model is, the more degrees of freedom it will have, so the machine resources consumption is generally unbearable to workstation.

For this simple plate structure, various finite element program, such as Cosmosm, Nastran, Adina, Ansys, etc, will solve all kinds of finite element analysis easily. The dynamics equation of the system can be written as a general form:

$$
[\mathrm{M}]\{\mathrm{u}\}+[\mathrm{C}]\{\mathrm{u}\}+[\mathrm{K}]\{\mathrm{u}\}=\{\mathrm{F}\}
$$

Thus, in this paper, the general finite element PATRANINASTRAN is used to implement the finite element analysis of the board. The analysis results are compared with the results of literature calculation by using the above modeling method [41].

Active vibration control usually includes two kinds of control methods: open-loop control and closed-loop control. At present, the research and application of closed-loop control is more active, 
whose basic idea is that appropriate system state or output feedback is utilized to generate certain control effect to change initiatively the zero closed loop, pole placement or structural parameters of the control structure so that the system meets the requirements of dynamic characteristics. Among them, the feedback control law design method basically uses the current achievements of the control theory, involving almost all branches of control theory, such as pole allocation, optimal control, adaptive control, robust control, fuzzy control, learning control and intelligent control method etc.

From the vibration theory, vibration of the system or structure can be placed in the modal space to study. Vibration in time domain with infinite degree of freedom system can usually use the approximate description of vibration in the low order degree of freedom system in the modal space. In this way, the vibration control of infinite degree of freedom system can be transformed into vibration control of a few modes in the modal space, i.e. control mode. This method is called mode control method, which is divided into modal coupling control method and the independent modal space control (IMSC) method. The latter can control independently the model that needs to be controlled. It does not affect other control mode and has the advantages of being designed easily. At present, it has become a mainstream method in model control.

The design of active vibration control system starts from constructing the system model, and then the simulation of model experiment will determine whether the designed control system meets the requirements of performance. The computer can be conveniently used to solve the mathematical model, so the computer is the main tool of mathematical simulation. The development of the computer technology, the high cost of the experiment and better active control technology enable the researchers to use computers to simulate the vibration control effect increasingly commonly, so the application of simulation technology has become inevitable.

The active plate model is made by directly pasting the piezoelectric plate on the substrate, shown in figure two and three. Piezoelectric actuators use 713A01 piezoelectric film produced by USA PCB of geometric size $\mathrm{L} * \mathrm{~W} * \mathrm{~T} 109 * 21.3 * 1.27 \mathrm{~mm}$, and the material is PZT5A. The piezoelectric sensor uses ICP piezoelectric strain sensor, model 740B02 produced by the PCB Pizotronics company. It is made of quartz with geometric size of $\mathrm{L} * \mathrm{~W} * \mathrm{~T} 15.2 * 5.1 * 1.8$. The substrate, piezoelectric sensors and actuators are glued with each other.

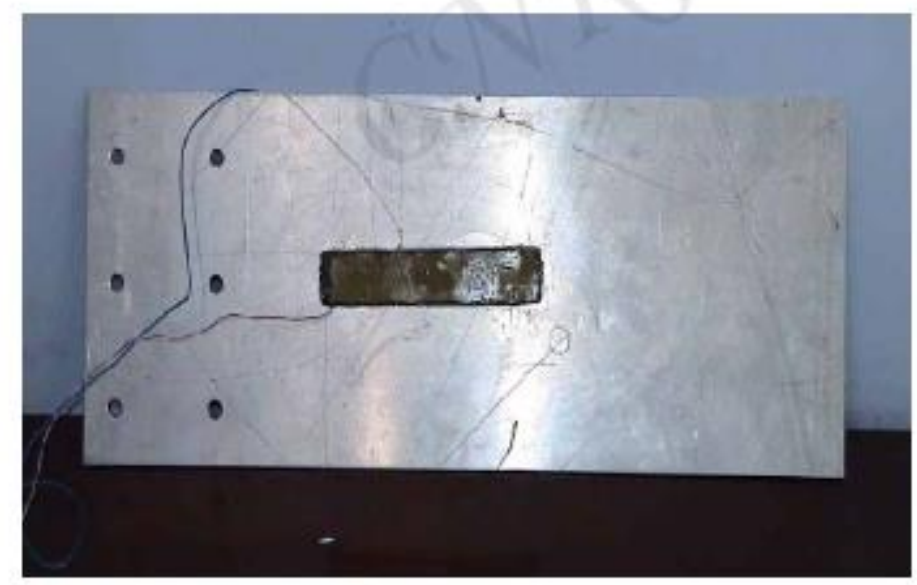

Fig. 1 paste the plate of the actuator 


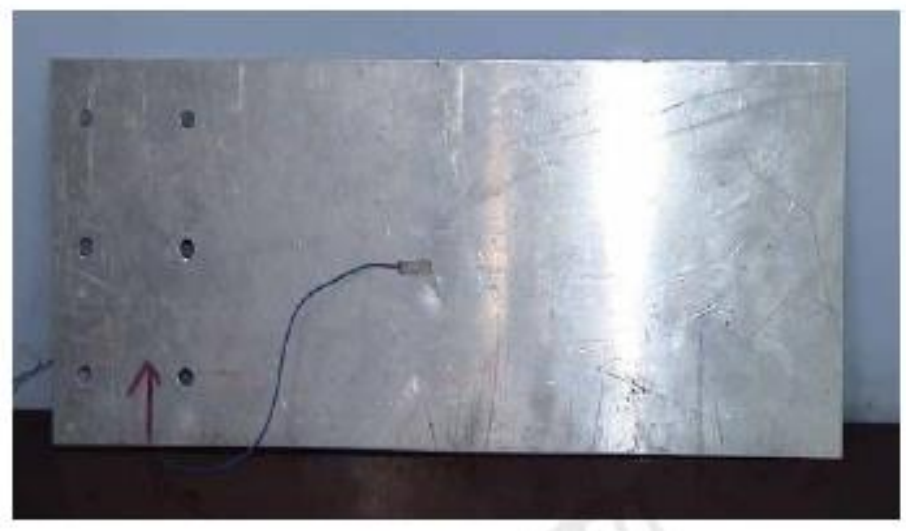

Fig. 2 paste the plate of the sensor

The experimental device of vibration control adopts xPC target real-time system based on MATLAB. The control system is shown in Fig. 1, including sensor, pre amplifier, A/D converter, D/A converter, computer, driver and actuator. The overall physical device is shown in Fig. 2, including 2 PC computer, 1 A/D data acquisition card with 8 channel PCL-818L (integrated D/A card), 1 AVC instruments 790 series power amplifier (piezoelectric drive power) and 1 PCI-481A sensor signal conditioner.

\section{Conclusion}

With the development of modern control, electronics, materials and other related disciplines, piezoelectric active control has become a hot research topic in recent years. Based on this background, the finite element method in practical engineering is used to study the active vibration control of piezoelectric active beam slab structure and has carried on the simulation and experiment. This paper has mainly done the following work:

1. In piezoelectric equations based on the Hamilton principle, assumed by Kirchhoff, with the neutral surface of the structure as reference, the 4-node piezoelectric element is used to establish the dynamic equation of active structure model.

2. Based on the research of piezoelectric element, the commercial finite element software PATRAN/NASTRAN is used to construct the active structure model to implement the finite element modeling and various kinetic analyses. It can be seen from the results that the modeling method is effective.

3. Apply coordinate transformation to the dynamic equations. Under the premise of fulfilling the demand of engineering precision, low order mode is used as the basis vectors to truncate the high order modal for decoupling.

4. In reference to the decoupled equations and the finite element analysis results, the proportional feedback control method and independent modal control method are studied based on LQR (two linear optimal control) and calculate the gain of active structural control to control the vibration of the structure.

5. In order to verify the validity of the vibration control method, finally we combine numerical examples and experiments to achieve responses before and after the active control of the structure and make the curve. The simulation results and experimental results show that using piezoelectric structure for active control of structural vibration suppression is effective. In this paper, modal analysis for the active structure and implement numerical simulation and experiments on the active 
vibration suppression are conducted, but there are still many problems needing to be explored and studied. The author thinks that further research work can be carried out in the following aspects:

1. Because the piezoelectric structure is a laminated structure and the accuracy of the model directly affects the accuracy of the calculation results, further research on the dynamic finite element modeling needs to be done;

2. Control method for various factors should be considered more maturely. There are many uncertain factors in the modeling process, such as the uncertainty of material physical parameter, the hypothesis of mathematical model, the error of model simplification and noise interruption, etc. Although the proportional feedback control method and independent model control method based on the LQR (two linear optimal control) are effective control methods, they are still needed further study;

3.Because of the hope of using low order model to replace the original controlled object model to reduce the amount of computation of designing the controller and to simplify the controller structure for the real-time implementation of control algorithm, more research on the reduced order model should be done;

4. Because the structure carried out in the experiment is too simple, further research in the experimental aspects needs to be done.

\section{Acknowledgements}

This work was financially supported by the key subject building project (vehicle engineering) of Jiangxi University of Technology.

\section{Reference}

[1] Bailey T, Ubbard J E. Distributed piezoelectric-polymer active vibration control of a cantilever beam[J]. Journal of Guidance, Control, and Dynamics, 1985, 8(5): 605-611.

[2] Balamurugan V, Narayanan S. Shell finite element for smart piezoelectric composite plate/shell structures and its application to the study of active vibration control[J]. Finite Elements in Analysis and Design, 2001, 37(9): 713-738.

[3] Fanson J L, Caughey T K. Positive position feedback control for large space structures[J]. AIAA journal, 1990, 28(4): 717-724.

[4] Vashist S K, Chhabra D. Optimal placement of piezoelectric actuators on plate structures for active vibration control using genetic algorithm[C]//SPIE Smart Structures and Materials+ Nondestructive Evaluation and Health Monitoring. International Society for Optics and Photonics, 2014: 905720-905720-14.

[5] Wu D, Huang L, Pan B, et al. Experimental study and numerical simulation of active vibration control of a highly flexible beam using piezoelectric intelligent material[J]. Aerospace Science and Technology, 2014, 37: 10-19.

[6] Nambu Y, Yamamoto S, Chiba M. A smart dynamic vibration absorber for suppressing the vibration of a string supported by flexible beams[J]. Smart Materials and Structures, 2014, 23(2): 025032. 
[7] Kwak M K, Yang D H. Dynamic modelling and active vibration control of a submerged rectangular plate equipped with piezoelectric sensors and actuators[J]. Journal of Fluids and Structures, 2015, 54: 848-867.

[8] Qiu Z, Ling D. Finite element modeling and robust vibration control of two-hinged plate using bonded piezoelectric sensors and actuators[J]. Acta Mechanica Solida Sinica, 2014, 27(2): 146-161.

[9] Omidi E, McCarty R, Mahmoodi S N. Implementation of modified positive velocity feedback controller for active vibration control in smart structures[C]//SPIE Smart Structures and Materials+ Nondestructive Evaluation and Health Monitoring. International Society for Optics and Photonics, 2014: 90571N-90571N-11.

[10]Parameswaran A P, Gangadharan K V. Parametric modeling and FPGA based real time active vibration control of a piezoelectric laminate cantilever beam at resonance[J]. Journal of Vibration and Control, 2014: 1077546313518818. 